\title{
UNA TEORÍA HOLISTA PARA LA MEDICINA
}

\section{A HOLISTIC THEORY FOR MEDICINE}

\author{
Lucía FEDERICO* \\ CEFHIC-UNQ/UNTREF
}

\begin{abstract}
Resumen: A partir del derrotero presentado en Saborido (2020), el presente artículo se propone profundizar en dos cuestiones claves que allí se desarrollan. En primer lugar, la discusión alrededor de la dicotomía naturalismo/holismo, con el fin de delimitar el campo de controversias. En segundo lugar, la distinción entre los aspectos científicos y tecnológicos de la medicina. El ahondar en ambos puntos nos permitirá plantear una alternativa metateórica de medicina holista, acompañando la propuesta del autor. Por último, presentaremos algunas conclusiones de carácter propositivo, que esperamos enriquezcan los aportes efectuados por el libro.
\end{abstract}

Palabras clave: medicina, filosofía, holismo, naturalismo, tecnología.

Авsтract: Based on the path presented in Saborido (2020), this article aims to delve into two key issues that are developed there. In the first place, the discussion about the naturalism/holism dichotomy, in order to delimit the field of controversies. Second, the distinction between the scientific and technological aspects of medicine. The analysis of both points will allow us to propose a meta-theoretical alternative of holistic medicine, accompanying the author's proposal. Finally, we will present some conclusions of a propositional nature, which we hope will enrich the contributions made by the book.

KEYwORDs: medicine, philosophy, holism, naturalism, technology.

\footnotetext{
${ }^{*}$ Docente de la Universidad Nacional de Quilmes. Centro de Estudios de Filosofía e Historia de la Ciencia. Bernal, Buenos Aires, Argentina. Coordinadora de Metodología de la Investigación de la Universidad Nacional de Tres de Febrero. Departamento de Ciencias Sociales. Sáenz Peña, Buenos Aires, Argentina. Mail: luciafed@hotmail.com. Este trabajo ha sido posible gracias al financiamiento de los proyectos: PUNQ 0990/19 (Universidad Nacional de Quilmes), PICT 2018-3454 (ANPCyT, Argentina), UNTREF 32/19 80120190100115TF y $80120190200069 T F$ (Universidad Nacional de Tres de Febrero).
} 


\section{Introducción}

El libro Filosofia de la medicina (Saborido, 2020) atraviesa gran parte de las discusiones que actualmente se dan en dicha área, interpelando a todo profesional de la salud. Incluso resulta relevante para aquel que simplemente quiera entender qué es esa cosa llamada «medicina». Los temas desarrollados son amplios y diversos, por lo que aquí sólo abordaremos algunos que resultan cruciales por la actualidad de los debates sobre ellos, tanto a nivel filosófico como disciplinar. La pregunta es por la medicina que tenemos y la que queremos, retomando la propuesta de la Organización Mundial de la Salud (OMS) de considerar a la salud de manera holista, contemplando sus dimensiones subjetivas/valorativas centradas en el bienestar. Por eso, el foco se centrará en pensar la caracterización de las unidades epistémicas de la medicina, de manera de entender dónde y de qué modo podría introducirse la cosmovisión holista en ellas. En este sentido evaluaremos de manera crítica el análisis realizado por Saborido en su mencionada obra.

La estructura del presente trabajo será la siguiente: en primer lugar, abordaremos, siguiendo a Saborido, los aspectos más importantes de la discusión alrededor de la dicotomía naturalismo/holismo de manera de delimitar el campo de controversias. En segundo lugar, procederemos a distinguir los aspectos científicos de la medicina de los aspectos tecnológicos, bajo el supuesto de que ciencia y tecnología poseen estructuras diferentes en sus unidades epistemológicas de análisis (e.e. sus "teorías» son distintas). Ello nos será de utilidad para caracterizar la forma que debería contemplar una teoría holista de la medicina, habida cuenta que, afirmaremos, dicha disciplina posee una naturaleza dual que posee algunos aspectos científicos y también algunos tecnológicos. Luego realizaremos la propuesta de teoría holista, a partir del marco metateórico presentado por Rolando García en su obra Sistemas Complejos (2007), acompañando la propuesta pragmatista del autor. Por último, presentaremos algunas conclusiones de carácter propositivo. 


\section{Naturalismo vs. holismo}

En filosofía de la medicina, la perspectiva de corte biologicista, y en algún sentido reduccionista (Saborido 2020:84), es denominada naturalismo.

En líneas generales el naturalismo, propuesto por Scadding (1968) y Boorse (1975), considera que el conocimiento que usa la medicina para tratar pacientes es conocimiento biológico. Para los naturalistas los médicos utilizan criterios científicos objetivos que les permiten distinguir entre un funcionamiento correcto y uno incorrecto de los órganos. Esto implica, por un lado, una distinción particular de los conceptos «salud-enfermedad», 1 pues para efectuar esta partición es necesario primero discernir entre funcionamientos biológicos normales y anormales. Por el otro, condiciona una práctica médica que presupone que hay algo como una normatividad biológica (Saborido, 2020:72), una forma natural correcta de funcionar.

La medicina, por tanto, procedería a partir de «modelos» biológicos, que dan cuenta del funcionamiento de los órganos, presuponiendo que hay algo así como «normas naturales» que determinan las actividades de nuestros cuerpos, de origen evolutivo (al ser adaptativas) y factibles de ser capturadas por dichos modelos. Más aún, para el normativismo vital (Saborido, 2020:78) es el propio cuerpo el que distingue entre estos estados e informa un funcionamiento anormal o patológico. Así, el dolor resulta un indicador de un funcionamiento anormal, «cierto estado negativo» (Saborido, 2020:81) como una hemorragia, un incremento de la presión arterial o la oclusión ateroesclerótica. En cambio, la ausencia de dolor indica un funcionamiento normal o saludable. Parafraseando a Leriche «La salud es el silencio de los órganos» (en Saborido, 2020:82).

Esta distinción no es menor, pues permite caracterizar la enfermedad atendiendo a las manifestaciones, los síntomas, de un funcionamiento o estructura

\footnotetext{
${ }^{1}$ Dejando de lado, por el momento, las cuestiones prácticas, no está de más señalar que una manera de distinguir «salud» de «enfermedad» puede ser mediante conceptos densos (thick concepts), distinción que presenta tanto una dimensión descriptiva como una valorativa (Saborido, 2020:24). Por lo pronto, y sin profundizar demasiado, diremos que vamos a distinguir enunciados capaces de ser valorados y enunciados valorativos. La partición de un conjunto de sujetos como sanos y enfermos, para nosotros, es una distinción conceptual propuesta por una teoría, aceptando que la estructuración lingüística del mundo conlleva necesariamente una dimensión valorativa.
} 
anormal, asentando el criterio clínico de diagnóstico común en la medicina de finales del siglo XVIII, señala el autor. Así, el nombre «tuberculosis» daba cuenta de la presencia de "tubérculos» en los pulmones vinculados a la hemoptisis (expectación de sangre). El desarrollo de la microbiología en el siglo XIX, que pone el foco en el agente causal, conlleva el desarrollo de otro tipo de criterio de clasificación, el etiológico. Así, a partir del descubrimiento de Koch de mycobacterium tuberculosis, es la presencia de la bacteria (el agente causal), y no la lesión anatómica, lo que constituye el criterio de diagnóstico de la tuberculosis. Con el posterior desarrollo de la fisiología, la categorización de algunas enfermedades se fundamentó en los procesos fisiológicos moleculares. Este enfoque, que se sustenta en una perspectiva mecanicista, presupone que cada enfermedad se corresponde ahora con un mecanismo patológico concreto en función del tipo de fallo biomédico que supone. La mayoría de las enfermedades son clasificadas en la actualidad siguiendo este criterio patogénico o mecanistico, afirma Saborido. Aquí se cuela un reduccionismo mecanicista, pues «el mecanicismo en filosofía de la medicina se suele vincular con una postura metodológica llamada reduccionismo» (2020:160). Sin abordar las distinciones canónicas sobre el reduccionismo (para ello ver Díez y Moulines, 1997), Saborido lo entiende, al igual que muchos filósofos de la medicina (ver p.e. Andersen, 2017), como la simplificación de la complejidad a uno o a pocos factores causales primarios, los mecanismos moleculares, para explicar, investigar e intervenir las enfermedades.

Aunque la manera de «clasificar» la enfermedad cambie con el tiempo, según lo visto, todos los criterios señalados se basan, en definitiva, en identificar los aspectos biológicos anómalos utilizando de forma más o menos refinada «clases naturales», lo que conlleva una ciencia que no apela a la subjetividad del paciente. Por el contrario, lo patológico se corresponde con valores objetivos a través de las respuestas del organismo (para una historia sobre ello, ver López Cerezo, 2008).

Si bien en la práctica los pacientes difícilmente se ajustan a los casos típicos, las clases naturales funcionan como una guía heurística, «una "idea” en términos platónicos para su diagnóstico» (Saborido, 2020:209), o «tipos ideales» weberianos. Como señala Saborido (2020:86) y otros (p.e. Sisti \& Caplan, 2017), es posible interpretar este estándar, aún alineado con los teóricos naturalistas, en términos estadísticos.

Desarrollado en los '70s por Boorse (1975, 1977, 1997, 2014), y aún vigente, como muestra acertadamente Saborido, el enfoque bioestadístico plantea un criterio objetivo de normalidad a partir de valores de referencia. Basándose en 
un determinismo evolutivo propone que lo sano «se corresponde con el comportamiento biológico de la mayoría de individuos de una clase de referencia, mientras que lo patológico no sería sino el comportamiento fuera de la norma» (Saborido, 2020:28).

Por último, una propuesta más difícil de encasillar, es la medicina basada en evidencia (MBE), que adquiere su nombre como tal en 1990 a partir de un grupo de epidemiólogos de la Universidad McMaster en Hamilton (Reiss \& Ankeny, 2016). Su idea central es que los datos que ofrece la observación del comportamiento estadístico de los organismos son más fiables que las inferencias que podamos extraer a partir del conocimiento de su biología. El foco está en el tratamiento: sin desmedro de la validez del conocimiento biomédico, la MBE "considera que lo que determina la fiabilidad de un cierto tratamiento es que haya demostrado su eficacia de forma reiterada y en condiciones que garanticen la objetividad, mediante ensayos clínicos» (Saborido, 2020:177). Pero estos ensayos no pretenden esclarecer las relaciones causales del fenómeno de estudio. Así, la MBE permite la toma de decisiones médicas aun cuando se desconoce el mecanismo de la patología, como en el síndrome de la muerte súbita del lactante donde, aún desconociendo la base biológica, los ensayos clínicos aleatorizados (ECA) muestran una correlación entre la postura en la que los bebés duermen y tal síndrome, además de señalar diferencias significativas entre sexo biológico y razas (2020:178).

Por ello, la MBE podría entenderse como «agnóstica» en cuanto a la causalidad, plantea Saborido (2020:180). En contra de un reduccionismo mecanicista, monocausal, la MBE se abre a la posibilidad de fenómenos producidos por «un conjunto de causas predisponentes» o de origen «multifactorial» (2020:182): las decisiones sobre la salud se toman en base a observaciones que van más allá de los aspectos puramente biológicos. En este punto, podríamos preguntarnos si realmente la MBE presenta un modelo de salud corrido del naturalismo. Sin embargo, nos alerta el autor, el problema de la $\mathrm{MBE}$ «es que, al mover el foco de la comprensión de la enfermedad a la cuestión de la eficiencia los tratamientos pasan por alto la cuestión acerca de qué condiciones deben ser consideradas como enfermedades y por qué» (2020:184). Aunque Saborido se enfoca en el tratamiento, la MBE es utilizada también en el diagnóstico médico. Entonces se vuelve al planteo inicial, ¿qué criterio de salud y enfermedad está concibiendo la $\mathrm{MBE}$ ? Varios autores han señalado la relevancia que aún tiene el conocimiento biomecanicista en los ECA (p.e. Solomon, 2015). Otros, el papel indispensable que cumplen tales explicaciones reduccionistas en las ciencias médicas (van 
Bouwel et al. 2011). Aunque existen en la literatura múltiples discusiones al respecto, es de nuestra opinión que, por el tipo de datos que usa, MBE se termina acercando aún al naturalismo, lo que no quiere decir que esto pueda cambiar en el futuro. Algo que creemos también intuye el autor cuando señala que la MBE es un «modelo que aboga por una postura muy diferente a la noción biomecánica del reduccionismo pero que también está muy lejos del enfoque social del holismo» (2020:171).

En síntesis, todas estas posiciones naturalistas (o aún cercanas a ella) tienen en común el uso del concepto «enfermedad» como un antónimo de «salud». Sin embargo es posible, atendiendo a algunas precisiones lingüísticas, una cosmovisión diferente (Reiss \& Ankeny, 2016, Saborido, 2020):

Desde la cirugía estética hasta la interrupción voluntaria del embarazo, la medicina parece tener como ideal algo más que el comportamiento fisiológico normal. La búsqueda del bienestar (subjetivo) y no de la normalidad (objetiva) es la razón de ser de gran parte de las terapias y medicamentos (Saborido, 2020:115).

Mientras que «enfermedad» entendida como «afección», se hace eco de una postura naturalista de la medicina, «enfermedad» entendida como «indisposición» y «malestar», encaja con una propuesta epistemológica distinta. Como menciona Hausman $(2012$, 2017), la salud es un componente del bienestar, pero no son la misma cosa.

En un giro de ciento ochenta grados, la Organización Mundial de la Salud (OMS) separa los conceptos de «salud» y «enfermedad» de forma tal que ahora la ausencia de enfermedad no implica salud. Es necesario algo más, «y no es precisamente poca cosa: es necesario que se dé un completo bienestar. Casi nada» (Saborido, 2020:56). El médico debe tener en cuenta todo un conjunto de factores diversos (biológicos, económicos, sociales, ideológicos y culturales) a la hora de tratar a un paciente exitosamente. Dicho sea de paso, estos factores si se hallan presentes en la mayoría de las teorías de la enfermería (Alligood, 2015).

En la filosofía de la medicina se llama holismo a la perspectiva integradora que propone que, para acceder al bienestar, se debe contar con un marco conceptual que dé cuenta de todos los aspectos que afectan a la salud y el bienestar humano. En 1987 Nordenfelt presentó una teoría holista, la cual propone que 
«una persona está sana si se siente bien y puede funcionar en su contexto social» (Saborido, 2020:109).

Ahora bien, el sufrimiento o falta de bienestar depende, en parte, de los valores y circunstancias vitales de cada individuo. Así, afirma Saborido, cada persona puede tener una experiencia muy distinta de una misma incapacidad, dependiendo de sus circunstancias personales, como también hay enfermedades que no conllevan dolor (p.e. ciertas enfermedades mentales).

El hecho de que ahora tal distinción esté sujeta, en parte, a la percepción de las personas en un contexto, lleva a que el holismo comparta algunas semejanzas con la posición epistemológica constructivista. "Lo que los constructivistas remarcan es que nuestras teorías, supuestos y resultados científicos son producto de nuestras mentes, que medimos los fenómenos del mundo a partir de nuestras propias características y que no son semblanzas fidedignas de la realidad» (2020:107).

Un constructivismo radical conlleva un relativismo peligroso, afirma el autor, que va desde un exceso de medicalización hasta los movimientos antivacunas, por lo que es necesario ponerle coto y dar con criterios para decir que no todo vale. Pero, como señala Saborido, la ciencia no es un conocimiento indiscutible sino un producto cultural conformado por una multitud de factores contingentes: «Si queremos conocer de verdad qué presupone e implica la distinción entre lo sano y lo enfermo, deberemos fijarnos de forma crítica en el conjunto de valores que conforman el paradigma de la medicina» (2020:109).

Sin embargo, no toda propuesta teórica es igualmente aceptada por la comunidad científica. Ese ajuste/aceptación está vinculado con el constante contraste de la teoría con los datos intersubjetivamente aceptados. Sin desconocer el enorme éxito que tiene la medicina podemos pensar que: «la postura más razonable sea asumir un enfoque híbrido que recoja tanto los aspectos subjetivos como los objetivos que pueden servir para distinguir lo sano y lo enfermo, y que esté abierto a la crítica y revisión constantes tanto por parte de la ciencia como de la ética y la política» (2020:121). 


\section{Ciencia vs. tecnología}

Frónesis es un término de base moral que retoma Saborido (2020) de la filosofía de la medicina para establecer tres dimensiones relevantes en dicha disciplina: la teórica, la práctica, y la normativa. Aristóteles separa sofia, el conocimiento teórico (nous y episteme), de la frónesis, que involucra, además de la habilidad práctica para alcanzar un fin (techné), la capacidad para reflexionar al respecto y así determinar «buenos fines». Por ello frónesis o "sabiduría práctica», determinada por una dimensión político-social y socio-cultural o contextual (Blanco Carrero, 2018), se relaciona muchas veces con la «prudencia» o la «conciencia».

Un sujeto prudente, señala Saborido, es alguien que tiene conocimiento teórico del mundo, la capacidad para actuar en consecuencia y de discernir racionalmente sobre sus acciones previendo si sus consecuencias pueden considerarse deseables. Así debería actuar un médico: bajo una moral virtuosa en el sentido aristotélico, para lo cual no solamente tiene que tener en cuenta los valores de los pacientes, sino también los propios. «Los médicos no se limitan simplemente a describir y explicar la realidad, sino a modificarla para que se ajuste a un ideal predeterminado» (2020:153). Ello involucra una dimensión moral.

Saborido propone entonces que la medicina tiene un aspecto científico, teórico, «lo más exhaustivo posible sobre la realidad biológica» (2020:153) y si bien se sustenta en ésta, no es reducible a ella pues difiere en cuanto a sus objetivos:

[S]i la medicina solo fuera conocimiento teórico aplicado, quizá pudiera reducirse a la biología o incluso considerarse un conglomerado de ciencias biológicas [...]. La medicina no tiene como objetivo principal simplemente describir una parte de nuestro mundo (en este caso, nuestros propios cuerpos), sino transformarlo para arreglarlo o mejorarlo. Es por ello que la medicina es inherentemente normativa (2020:153).

Esta diferencia con la biología radica en la techné (el arte de curar), en una destreza técnica «sofisticada que les permiten diseñar o utilizar fármacos, herramientas y métodos terapéuticos con los que tratar a los pacientes» (2020:153) y una práctica médica adecuada, ética, en beneficio de los pacientes. La techné más el aspecto ético implica entonces frónesis. 
Afirma Saborido que no es posible separar la dimensión práctica de la teórica, pues ambas son cruciales para comprender la medicina. La frónesis incluso juega un papel fundamental a la hora de determinar el par sano/enfermo. Creemos sin embargo que distinguir ambas dimensiones puede arrojar luz sobre su naturaleza y sobre la de otra disciplina de la salud como la enfermería. Para ello abrevaremos en la filosofía de la tecnología.

En primer lugar, la distinción clásica entre técnica y tecnología propone que, mientras:

[La] técnica es esencialmente la aplicación de un cierto know-how [saber-cómo], constituido a través de la acumulación y transmisión de experiencias concretas [...] sin un knowing why [saber-porqué] [...]. El sufijo "logía" de la palabra tecnología, nos invita a aprovechar el aspecto teórico que usualmente se encuentra atado con su uso [...] que sirve para indicar la presencia de algún tipo de dimensión científica o teórica (Agazzi, 1998:2).

La medicina, por tanto, involucra aspectos tecnológicos, pero ¿se trata sólo de una tecnología? Echeverría (1995), propone cuatro contextos científicos para enriquecer la distinción clásica de los contextos en la dinámica científica (de descubrimiento y de justificación) introducida por Hans Reichenbach en su libro Experience and Prediction (1938): el contexto de educación, de innovación, de validación y de aplicación. Aunque todos relevantes, a los fines del artículo nos concentraremos en el último. No es lo mismo ni elaborar ni presentar una teoría que aplicarla para la resolución de cuestiones concretas: «esta última tarea suele implicar el uso de artefactos tecnológicos que implementan a las teorías científicas y cuya construcción está regida por valores distintos de los que priman en la investigación cognoscitiva" (1995:59). Durante esta etapa de aplicación el criterio de valoración principal es «el it works (funciona), pero cabe aplicar otros muchos: desde la rentabilidad económica hasta la utilidad social» (1995:64).

Precisando aún más la distinción, Giri (2017) propone que «la ciencia posee una intencionalidad de búsqueda de una versión ontológicamente debilitada de la verdad que no posee la tecnología, cuya intencionalidad es más pragmática; básicamente, la búsqueda de soluciones a problemas prácticos. Así, la eficacia es un valor mucho más importante para la tecnología que el acercamiento a la verdad de una teoría científica» (2017:56). 
Entonces, si consideramos la ciencia (Díez y Moulines, 1997) como un conjunto de afirmaciones sobre un determinado ámbito de la realidad, que utiliza, en la búsqueda de conocimiento, al menos algún método denominado científico y sus unidades epistémicas son las teorías (o en los distintos sentidos que la filosofía de las ciencias las ha entendido, como matrices disciplinares, paradigmas, modelos, mecanismos, etc.), queda entonces buscar cuáles son las unidades epistémicas propias de la medicina. ${ }^{2}$

Lorenzano $(1977,2007)$ señala la dificultad de la búsqueda mencionada pues la percepción habitual de la medicina es la de la actividad que realizan los médicos. Por ello, o bien la medicina es una tecnología o es simplemente conocimiento biológico o, quizás una conjunción de ambos (prejuicios) resultando biología aplicada.

Aunque la salud y la enfermedad humanas son el territorio exclusivo de la investigación médica, la disciplina claramente se apoya en el conocimiento biológico. No por ello se puede decir que los enunciados distinguibles como propios de la medicina se «deducen» de los enunciados propios de la biología. Caso contrario la medicina sería reductible, en un sentido clásico, a la biología, algo que niegan tanto Lorenzano (2007) como Saborido (2020), aunque por razones distintas.

La ciencia médica se ocupa de responder preguntas como «¿qué es la enfermedad?», y «¿cómo se enferma una persona?». La práctica médica, en cambio, se ocupa de responder "¿cómo se cura la enfermedad?», lo que implica una terapéutica. Lorenzano (2007) decide encarar la elucidación del núcleo de la investigación médica, que llama la «clínica médica». Esta estructura teórica central de la medicina sería la parte científica, equivalente a la física, mientras que la terapéutica, parte práctica, tecnológica, sería equivalente a la ingeniería. En la clínica médica se reconocen tanto el plano semiológico (los signos físicos que presentan los pacientes), como el plano anatomofisiopatológico (las lesiones características de los distintos órganos). La teoría clínica, entonces, sería una construcción conceptual en la cual se conectan síntomas y signos específicos con alteraciones específicas para una enfermedad. Además, la clínica médica cuenta

2 Saborido (2020) menciona: epidemiología, la teoría microbiana, teoría médica tradicional (para el enfoque biologicista) y aquella que aparece siempre como característica de la medicina, la teoría de los humores, como ejemplos de teorías que no serían meramente biológicas debido a su carácter práctico, implicando «reglas tecnológicas» en el sentido de Bunge (1966). 
la evolución conjunta de alteraciones orgánicas, signos y síntomas de la enfermedad, es decir, de cómo las evoluciones anatomofisiopatológicas determinan la evolución de la enfermedad, lo que en medicina llaman la evolución clínica. La teoría propone así un enunciado general, por medio del cual se conoce cómo evoluciona el sistema, integrado por los elementos que se encuentran en cada plano (el semiótico y el anatomofisiopatológico), y las relaciones que se entablan entre ellos. Este enunciado a su vez establece una relación causal: el plano profundo, de lesiones anatomofisiopatológicas (desde el siglo XIX, cuando Virchow y Bernard sientan las bases de la fisiopatología), es la causa de los signos y síntomas. Así, la teoría explica qué es la enfermedad y cuál es la causa, dando cuenta del aspecto etiológico.

A medida que avanza la biología, los mecanismos anatomofisiopatológicos cambian sus fundamentos en mecanismos más profundos, genéticos y/o moleculares. Ahora las lesiones anatomofisiopatológicas son explicadas por la falla de algún mecanismo a nivel genético o molecular. Tales mecanismos en ocasiones reemplazan por completo a las primeras, sus evoluciones determinan la dinámica de la enfermedad, entendida como co-evolución de alteraciones orgánicas, signos y síntomas.

En este sentido la medicina se apoyaba en la biología, a la que presupone (de manera similar a como la astronomía presupone y se apoya en la física) pero responde a problemas distintos. Vista así, la teoría clínica (Lorenzano muchas veces habla de ella como una teoría general que abarca incluso a las enfermedades infecciosas y otras veces las distingue, como teoría de las enfermedades y teoría de las enfermedades infecciosas) ${ }^{3}$ puede caracterizarse como una ciencia básica, similar a la física o la química.

Esta estructura teórica es aquella que captura las apreciaciones de Saborido cuando afirma que los diferentes criterios (clínicos, etiológicos y mecanísticos) pueden coexistir dentro de un mismo marco teórico: «Es en este sentido en el que podemos decir que se da un pluralismo integrador en medicina» (2020:208). ${ }^{4}$

\footnotetext{
3 Las enfermedades infecciosas son explicadas bajo una estructura teórica similar, la diferencia radica en que en este caso las lesiones anatomofisiopatológicas son causadas por un agente biológico externo.

4 A diferencia de nuestra propuesta, Saborido sugiere un "pluralismo integrador pragmatista» (2020:215).
} 
A partir de estas estructuras teóricas el médico puede efectuar un pronóstico médico, equivalente a la predicción científica: si el conjunto de signos que presenta el enfermo es el que ahora se describe, puede decirse que el futuro de ese enfermo será tal o cual. Por otro lado, diagnosticar que un paciente padece una enfermedad dada, consiste en afirmar que es un ejemplar, en el sentido de Kuhn (1971), de la teoría. Es decir, que el paciente es un "caso del mismo tipo" de una matriz disciplinar, en el sentido de un nominalismo de semejanzas de corte wittgensteiniano, ya que ningún paciente es igual a otro. Luego de este proceso se pueden comenzar a urdir mecanismos de prevención y de curación de la enfermedad, lo que lleva al tratamiento médico, de carácter tecnológico.

Para la práctica médica este punto es crucial, pues a la hora de aplicar un tratamiento concreto lo que se espera del mismo no es un incremento de la capacidad cognoscitiva de la teoría (que puede verse como un producto secundario del proceso) sino que funcione eficaz y eficientemente: "puede ser aplicada para modificar, transformar y mejorar el medio, el entorno, el mundo o la realidad» (Echeverría, 1995:64). Aquí es clara la dimensión valorativa y la presencia de enunciados nomopragmáticos (Bunge, 1966). Además, para su creación, los tecnólogos utilizaron alguna forma más o menos organizada de heurística ingenieril (Giri, 2017), proceso cognitivamente distinto al método científico.

Lorenzano (2007) menciona además otra teoría, la medicina social o sanitarista. En ella, los hechos sociales (que incluyen hechos antropológicos y psicológicos) determinan hechos biológicos, y éstos determinan la enfermedad. A partir de estas intuiciones y distinguidos los aspectos epistémicos de los tecnológicos bajo la premisa de que la medicina es distinta que la biología por sus teorías, es momento de preguntarse cómo sería, o debería ser, una teoría de la medicina que capture el sentido de «salud» señalado por la OMS.

\section{Un holismo para la medicina}

A la hora de pensar una alternativa holista para la medicina, Saborido apela a una visión pragmatista de la ciencia. De forma sucinta, el pragmatismo de Pierce, James y Dewey propone que los conceptos de las teorías, aquellos con los que se interpreta y se interviene la realidad, dependen de la interacción con ella. La noción de "verdad», de "adecuación empírica de la teoría», queda ahora 
supeditada a su utilidad: «Decidir si un término, una teoría o una clasificación son válidas dependerá de su valor práctico con respecto a las metas epistémicas de aquellos que los utilizan» (2020:211). Para el pragmatismo, las categorías propuestas por la ciencia son herramientas metodológicas y no clases naturales. Así, el cambio conceptual teórico se explica atendiendo «al papel que los conceptos juegan con respecto a las prácticas científicas en cada momento» (2020:211). El progreso científico dependerá del mayor o menor grado de proximidad a los fines propuestos por la ciencia. Se progresa cuando se producen teorías mejores y más fiables para intervenir el mundo.

En medicina, señala Saborido, «la categorización de ciertos estados como enfermedades debe basarse principalmente en consideraciones pragmáticas» (2020:210). Según esta propuesta, cuando el profesional de salud, el paciente y la sociedad no consideran de la misma manera qué es una enfermedad, es hora de revisar las categorías de las teorías, pues los conceptos son inseparables de las acciones y si lo que se busca es una medicina holista la práctica médica debe contemplar estos enfoques. Si en algún momento «nociones como "esquizofrenia" o "trastorno bipolar" dejan de servir al ejercicio con frónesis de la psiquiatría, entonces estos conceptos deben ser eliminados o sustituidos, del mismo modo que lo fueron categorías como "histeria” o "drapetomanía” (2000:244).

Se explicita nuevamente la relevancia de la frónesis incluso en la distinción de los conceptos. Si hay una demanda social de una práctica médica que contemple enfoques diversos como el del paciente y el de la sociedad misma, los conceptos de las teorías medicas deberán cambiar para satisfacerla. «Lo importante es que la clasificación sea lo más útil posible para los médicos facilitando los diagnósticos y una mejor comprensión de las enfermedades» (2000:212-213).

Si bien creemos que el enfoque pragmatista es interesante y pertinente para analizar la medicina, también creemos que un análisis centrado en teorías no sólo lo complementa, sino que permitirá arrojar luz sobre el holismo que buscamos. Por ello, resulta inspirador recorrer las páginas del clásico Sistemas complejos de Rolando García (2007) a la hora de imaginar la estructura de una teoría holista que dé cuenta de la salud humana caracterizada por su complejidad. El modelo del médico clínico generalista, el recorte disciplinar del médico especialista y la relevancia del diagnóstico médico, son menciones recurrentes del libro. A partir 
de la propuesta de García (2007), identificaremos metateóricamente como debería ser una teoría holista. ${ }^{5}$

El primer paso para pensar cómo debería ser una teoría holista de la salud o «salud sistémica», es preguntarse cómo se debe reconocer el objeto de estudio. Si este no es reducible a la yuxtaposición de situaciones o fenómenos que pertenezcan al dominio exclusivo de una disciplina como la biología, entonces es un sistema que puede entenderse como un "complejo» (2007:93).

Puesto que todo estudio supone necesariamente un recorte de la realidad, el primer problema subyace en la manera en que se fijan los límites del sistema, de forma tal de que no se desnaturalice ni pierda sus características relevantes, algo que parece ocurrir con los enfoques biologicistas de la salud al sólo capturar una parte de la totalidad del fenómeno objeto. El sistema es una totalidad organizada conformada por distintos elementos que configuran una estructura dinámica. El recorte para su análisis debe respetar dicha estructura. En este caso el sistema objeto es la salud sistémica. La estructura es un conjunto de elementos que se relacionan en virtud de sus propiedades en un sistema organizado que se mantiene en condiciones estacionarias mediante procesos dinámicos de regulación homeostática. El recorte del sistema debe incluir las relaciones más significativas de sus elementos, los cuales pueden ser a su vez (sub)sistemas que interactúan entre sí y que pueden ser analizados de forma independiente en otro nivel de estudio. Así entendida, la salud sistémica está conformada al menos por: el nivel biológico, el nivel psicológico y el nivel sociológico. Cada nivel presenta sus propias propiedades pero en salud lo que importa es el emergente de la interacción entre los niveles y las propiedades del sistema.

Ahora bien, un modelo o teoría es una construcción del sistema de forma tal que pueda explicar su funcionamiento, dando cuenta de los hechos observados. La reconstrucción teórica del sistema necesita la modelización de cada subsistema, la velocidad de desarrollo de los procesos generados por los subsistemas, las interrelaciones causales, los valores críticos que introduzcan inestabilidad potencial al sistema y las posibles fluctuaciones que pudieran desestabilizarlo. Esta reconstrucción debe completarse con la evolución del sistema, es decir, con un análisis sincrónico de éste que difiera de la dinámica de sus componentes

5 Acompañando el espíritu de la medicina de sistemas (ver Vogt, Hofmann \& Getz, 2016), pero ahora pensando una teoría propiamente médica, es decir, sin el biologicisimo implícito de dicha propuesta. 
(para mayor detalle mirar García, 2007:103-104). Las trasformaciones sistémicas responden así a una "ley muy general: la evolución no lineal, con discontinuidades estructurales, que procede por sucesivas reorganizaciones» (2007:75-76).

Los sistemas complejos son abiertos (interactúan con el entorno), y cuando las condiciones de contorno (los flujos entre sistemas y entorno) sufren pequeńas fluctuaciones el sistema se mantiene estacionario pues las relaciones entre los elementos fluctúan sin que se modifique la estructura. Sin embargo, si las perturbaciones (exógenas o endógenas) no pueden ser amortiguadas por el sistema, ocurre una irrupción con una posterior evolución. La estructura reacciona cambiando, se reorganiza hasta lograr una nueva estabilidad, y el sistema vuelve a ser estacionario pero con una nueva estructura. La consideración holista de la salud permite comprender cómo factores perturbadores en alguno de los niveles (biológico, psicológico, social) genera, dependiendo de la resilencia del paciente (la cual depende de las propiedades de cada uno de sus niveles) puede generar como emergente una respuesta en el que puede estabilizarse sólo (puede «autocuidarse», en el sentido de Orem, 2001) o se desestabiliza de modo tal de requerir la intervención del sistema de salud (o en el peor de los casos, morir).

Souza et al. (2004) estudian la influencia de distintos factores en la capacidad de autocuidado de una muestra de pacientes externados con diabetes mellitus. El hecho de estar externados demuestra que la salud de los pacientes conforma un sistema estacionario estable: aunque padecen la enfermedad, se encuentran listos para volver a sus hogares. Sin embargo, los autores demuestran que variables de nivel biológico (p.e. la raza, el tipo de diabetes), psicológico (p.e. la autopercepción de su salud) y social (p.e. el nivel de educación, el poseer una red de contención social) son cruciales para estimar la resiliencia del sistema en términos de capacidad de autocuidado. Esto explica el fenómeno de la salud de los pacientes diabéticos, pero también señala estrategias de intervención: queda claro que las acciones relegadas únicamente al nivel biológico serán como mínimo inefectivas.

La modelización de un sistema de estas características parece ciertamente muy compleja. Sin embargo gran parte de la modelización se ve allanada por las teorías disciplinares de cada subsistema. Cada nivel de organización es semiautónomo y en cada uno rigen dinámicas específicas. Estos niveles se han estudiado de forma desacoplada y las teorías formuladas sobre ellas presentan cierta estabilidad. Lo que propone García (2007) es su acoplamiento vía una actividad cognitiva interdisciplinaria. La articulación de las diversas teorías y disciplinas se logra en un enfoque integrado bajo una «metodología particular de investigación 
que concibe el problema desde la peculiar perspectiva de los sistemas complejos» (2007:91-92). Sin precisiones reconstructivas, por cuestiones de espacio, podríamos señalar que la forma en que algunos gobiernos afrontaron la pandemia mundial COVID-19, en una estrecha vinculación con la OMS, es un ejemplo de un enfoque de este tipo: se combinan acciones sobre el nivel biológico (p.e. diseño de tests, terapias y vacunas), acciones de nivel social (p.e. políticas de aislamiento, de búsqueda de posibles infectados, campañas de concientización, un sistema educativo virtualizado, subsidios a personas que han perdido su capacidad de generar ingresos económicos por el aislamiento) y psicológico (p.e. campañas de autocuidado). Otro ejemplo en el mismo sentido es el de la atención a víctimas de violencia de género, que implica (en condiciones ideales) acciones que no se limitan únicamente a la atención de las heridas sino también al apoyo psicológico y acciones sobre el entorno social inmediato. ${ }^{6}$

\section{Conclusiones}

Filosofía de la medicina, nos advierte Saborido (2020), pretende utilizar la filosofía de la ciencia contemporánea para ayudar a entender mejor lo que presupone e implica la práctica médica. La idea de que el médico debe tener en cuenta un conjunto de factores diversos como los biológicos, económicos, sociales, ideológicos y culturales a la hora de construir una teoría o tratar a un paciente con éxito, no es una cuestión menor. Tampoco el discernir «si la medicina es una ciencia o es una técnica, un arte, que depende más de la habilidad del profesional que de los conocimientos puramente teóricos» (2020:30). En parte, porque nos permite pensar qué medicina queremos, y en parte porque nos permite tomar medidas para lograrlo.

Una teoría holista de la salud como la que nos proponemos (y entendemos compatible con la mirada de la OMS) permitiría resaltar la importancia de incluir aspectos psicológicos y sociológicos en la formación de los médicos. Esto no implica que el profesional deba ser a su vez psicólogo y sociólogo, sino que debe ser capaz de comprender el lenguaje de estos profesionales para trabajar

6 El modelo aquí propuesto comparte cierta esencia con algunas propuestas anti-reduccionistas mencionadas por Mitchell (2002) y Andersen (2017), que no desarrollaremos por cuestiones de espacio. 
junto a ellos en equipos interdisciplinarios o transdisciplinarios (y no multidisciplinarios, pues la idea es que exista un enfoque y lenguaje unificado y no un trabajo "parcelado» donde cada cual habla de lo que sabe de manera inconexa). Esto permitiría enfoques más completos sobre el fenómeno complejo de la salud, no limitados únicamente al trabajo clínico (de carácter netamente fisiológico).

Por otra parte, insistir en la existencia de una dimensión científica en la medicina también sugiere estrategias para modificar las currículas de las facultades de medicina. Si bien por cuestiones de espacio escapa a este trabajo demostrarlo empíricamente, entendemos que en la mayoría de las Universidades se enfatiza más el aspecto tecnológico de la medicina, lo que resulta en un enfoque profesionalista que desemboca predominantemente (pero no únicamente) en un profesional «de consultorio». El énfasis en la dimensión científica llevaría a incrementar el entrenamiento en metodologías de investigación clínica, en trabajo de laboratorio, y en la conformación de equipos interdisciplinarios como los que sugerimos basándonos en la propuesta de García. De este modo podrían despertarse más vocaciones de investigación además de las netamente clínicas (cuya importancia de ningún modo negamos), lo que podría llevar eventualmente a una aceleración en la obtención de resultados científicamente relevantes alrededor de distintos problemas acuciantes para los sistemas de salud.

Filosofía de la medicina atraviesa una multiplicidad de discusiones cruciales que se plantean alrededor de la ciencia de la salud. Recomendamos enfáticamente abrevar en él a todo aquel que desee encontrar una mirada clara y profunda sobre estas problemáticas.

\section{Agradecimientos}

Al Dr. Ing. Leandro Giri, por su invaluable ayuda y acompañamiento constante. 


\section{Bibliografía}

Agazzi, E. (1998). "From Technique to Technology: the Role of Modern Science”, Phil \& Tech, 4(2), 1-9.

Alligood, M. E. (2015). Modelos y teorías de la enfermería. Barcelona: Elsevier.

Andersen, H. K. (2017). "Reductionism in the Biomedical Sciences". In Solomon, M., Simon J.R., H. Kincaid (eds.), The Routledge Companion to Philosophy of Medicine, New York: Routledge-Taylor \& Francis, 81-89.

Blanco Carrero, E. E. (2018). "El concepto de 'phrónesis': desde Aristóteles hasta Francisco de Suárez", Mutatis Mutandis: Revista Internacional de Filosofía, 10, 93-116.

Boorse, C. (1975). "On the distinction between disease and illness", Philosophy \& Public Affairs, 5(1), 49-68.

— (1977). "Health as a theoretical concept", Philosophy of Science, 44(4), 542-573.

- (1997). "A rebuttal on health". In Humber, J.M., R. F. Almeder (eds.), What is disease? Towtowa, NJ: Humana Press, 3-134.

— (2014). "A second rebuttal on health", Journal of Medicine and Philosophy, 39, 683724 .

Bunge, M. (1966). "Technology as Applied Science”, Technology and Culture, 7(3), 329-347.

Díez, J. y Moulines, U. (1997). Fundamentos de filosofía de la ciencia, Barcelona: Ariel.

Echeverría, J. (1995). Filosofía de la Ciencia, Madrid: Ediciones Akal.

García, R. (2007). Sistemas complejos, Barcelona: Gedisa.

Giri, L. (2017). "Modelización, predicción y valores sociales”. En Tula Molina,F., H.G. Giuliano (eds.), El riesgo de que todo funcione. Para una evaluación amplia de la tecnología, Buenos Aires: Nueva Librería, 37-60.

Hausman, D. (2012). Preference, Value, Choice and Welfare, Cambridge: Cambridge University Press.

— (2017). "Health and Well-Being". In Solomon, M., Simon J.R., H. Kincaid (eds.), The Routledge Companion to Philosophy of Medicine, New York: Routledge-Taylor \& Francis, 27-35.

Kunn, Th. S. (1971). La estructura de las revoluciones cientificas, México D.F.: FCE.

Lorenzano, C. J. (1977). "Análisis metodológico de una ciencia empírica: la medicina”, Revista de filosofía DIÁNOIA, [S.1.], 23(23), 124-136. 
- (2007). "El devenir de la medicina: una mirada epistemológica”, ponencia en la mesa Homenaje a los Maestros de la Filosofía Argentina, Actas del Congreso Internacional Extraordinario de Filosofía, San Juan, Argentina.

López Cerezo, J. A. (2008). El triunfo de la antisepsia. Un ensayo en filosofía naturalista de la ciencia, México D.F.: FCE.

Mitchell, S. D. (2002). "Integrative pluralism”, Biology and Philosophy, I7(I), 55-70.

Orem, D. (2001). Nursing: Concepts of Practice, St. Louis: Mosby.

Reichenbach H. (1938). Experience and prediction, an analysis of the foundations and the structure of knowledge. University of Chicago Press.

Reiss, J., Ankeny, R.A. (2016). "Philosophy of Medicine", The Stanford Encyclopedia of Philosophy, E.N. Zalta (ed.). https://plato.stanford.edu/archives/sum2016/entries/ medicine/.

Saborido, C. (2020). Filosofía de la medicina, Madrid: Tecnos.

Scadding, J. G. (1968). “The clinician and the computer”, The Lancet, 291, 139-140.

Sisti, D., Caplan, A. L. (2017). “The Concept of Disease”. In Solomon, M., Simon J.R., H. Kincaid (eds.), The Routledge Companion to Philosophy of Medicine, New York: Routledge-Taylor \& Francis, 5-15.

Solomon, M. (2015). Making Medical Knowledge, Oxford: Oxford University Press.

Sousa, V. D., Zauszniewski, J. A., Musil, C.M., McDonald, P. E., Milligan, S. E. (2004). "Testing a Conceptual Framework for Diabetes Self-Care Management", Research and Theory for Nursing Practice: An International Journal, 18(4), 293-316.

Van Bouwel, J., Weber, E., de Vreese, L. (2011). "Indispensability Arguments in Favour of Reductive Explanations", Journal for General Philosophy of Science, 42, $33-46$.

Vogt, H., Hofmann, B., Getz, L. (2016). "The new holism: P4 systems medicine and the medicalization of health and life itself", Med Health Care and Philos, 19, 307-323.

Este trabajo se encuentra bajo una licencia de Creative Commons Reconocimiento-

NoComercial-SinObraDerivada 4.0 